\title{
Primary structure of CuA and CuB domain of Ferula communis polyphenol oxidase
}

\author{
V. Carracoi, E.Atzori,A. Rescigno', E. Sanjust',A. Padiglia \\ Department of Sciences Applied in Biosystems \\ 'Department of Biomedical Sciences and Technologies, University of Cagliari, Italy
}

Polyphenol oxidases (PPOs; EC I.14.18.1, 1.10.3.1) are ubiquitous plant enzymes capable of catalyzing the oxidation of o-diphenols to their corresponding o-quinones [I]. Although they are nearly ubiquitous among plants, the exact roles they play in plant growth, development, and physiology are not entirely clear. In this work we isolate a cDNA fragment encoding the catalytic sites of polyphenol oxidase from Ferula communis with the aim of understanding its primary structure, function and molecular features compared with other PPOs from various plants and fungi. Total RNA was isolated from Ferula communis leaves using the TRI Reagent RNA isolation reagent (Sigma) according to the manufacturer's instructions. To obtain cDNAs, Ferula communis RNAs were reverse transcribed with an oligo-dT primer using an enhanced avian myeloblastosis virus reverse transcriptase enzyme (Sigma). Oligonucleotide primers for PPO were designed using the consensus degenerate hybrid oligonucleotide primer (CODEHOP) strategy [2], starting from the alignment of multiple PPOs sequences from different plant sources. Eight sequences were chosen from the GenBank database and aligned using ClustalW (http://www.ebi.ac.uk/clustalw), and then cut into blocks using the Block Marker software (http://blocks.fhcrc.org/blocks/). Primers were designed using the default parameters at the CODEHOP server (http://blocks.fhcrc.org/codehop.html). The sense primer was used in different PCR experiments in combination with each of the three selected antisense primers. PCR was performed in a solution containing $1.5 \mathrm{mM}$ $\mathrm{MgCl}_{2}, 100 \mathrm{mM}$ Tris $\mathrm{HCl}, \mathrm{pH} 8.3,50 \mathrm{mM} \mathrm{KCl}, 200 \mathrm{mM}$ of dNTP mix, I mM sense primer, I mM antisense primer, $50 \mathrm{ng}$ of Euphorbia cDNA, and I-3 units of Jump Start AccuTaq LA DNA Polymerase Mix (Sigma, St. Louis, MO). Thermal cycles of amplification were carried out in a Personal Eppendorf Mastercycler (Eppendorf, Hamburg, Germany) using slightly different programs. CDNA sequencing was performed by SharDNA (Cagliari, Italy) and translation of nucleotide sequences was done by the ExPASy translate software (http://ca.expasy.org/).Analysis of the F. communis PPO sequence was performed through bioinformatics programs (http://elm.eu.org/; http://cbs.dtu.dk/services/NetNGlyc/). Degenerate PCR primers corresponding to conserved domains of PPOs were designed with the aim of amplifying cDNA fragments encoding this PPO family in Ferula communis. The most efficient amplification was obtained with the pair of CODEHOP primers complementary to the motifs flanking the two active site, $\mathrm{CuA}$ and $\mathrm{CuB}$ domain, in all plant
PPOs. The product of expected length (approximately 650 bp) obtained by PCR experiments, was purified, and sequenced. BLAST searches of GeneBank revealed highly significant homology of this nucleotide sequence of Ferula cDNA fragment, to other plant PPO sequences. The deduced amino acid sequence of Ferula, was aligned with the well known Ipomea batatas PPO primary structure (accession number AJ006097), with the aim to obtain detailed molecular information. Numbers above the aligned sequences in figurel, correspond to numbering of amino acid residues in Ipomea PPO [3] The invariant amino acids in the aligned sequences are indicated by stars. The six histidine residues involved in copper binding $\mathrm{CuA}$ and $\mathrm{CuB}$, are indicated by thin and bold arrows respectively. The residue $E_{236}$ found in Ipomea PPO $\mathrm{CuB}$ (indicated by $\uparrow$ ), proposed to function as a general acid/base in diphenol oxidation, is conserved in the Ferula enzyme. Moreover, $\mathrm{Cys}_{92}$ described to form an unusual thioether bond with $\mathrm{H}_{109}$ (residues boxed in $\mathrm{CuA}$ domain) is also conserved. The sequence has been deposited in the GenBank database (accession NACL68094).

\section{References}

[I] Marusek C.M., Trobaugh N.M., Flurkey W.H., Inlow J.K. 2006. Comparative analysis of polyphenol oxidase from plant and fungal species. J. Inorg. Biochem. 100: 108-123.

[2] Rose T.M., Henikoff J.G., Henikoff S. 2003. Nucleic Acids Res. 31 : 3763-3766.

[3] Gerdemann C., Eicken C., Magrini A., Meyer H.E., Rompel A., Spener F., Krebs B. 200I. Isozymes of Ipomoeo batotos catechol oxidase difer in catalase-like activity. Biochim. Biophys. 1548:94105.

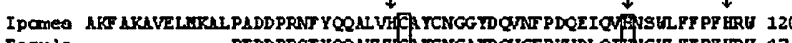
rerula - -

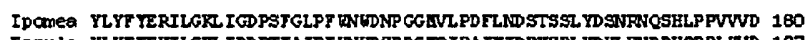

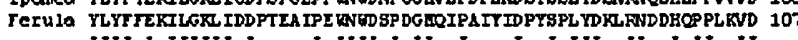
rerula YLY = I PCMCO LGNGUDTOUTDQOR ITDNLLL NYKONN TN LGTARLFLGRA YRAGD APSPGAGSIETSPH 240

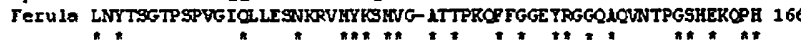
" *

IPCME IPI HRTVGDPRNTNNED MGNT YSAGRD IAP YCHESNVDRETT THOOLAGKPRKRDYTDSD 300 Ferulo TEUHIUTGDPNOIHGEDHGRFYSAGRDP IF YSHHUNDRMUI IUKTLPGKI - .."

Figure - Alignment of amino acid sequences of Ipomea batatas and Ferula communis PPO. 